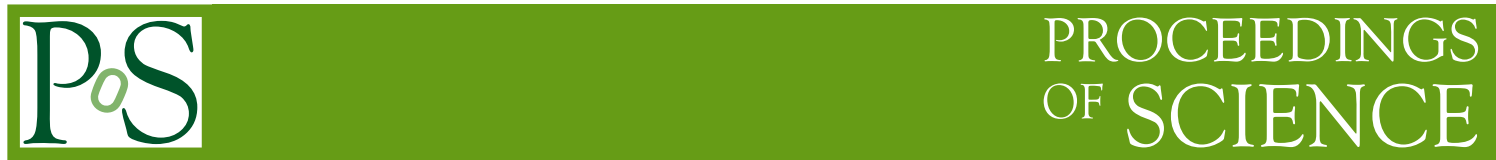

\title{
Search for SUSY in final states with photons at the Tevatron
}

\section{Andrey Loginov*†}

Yale University, Physics Department, New Haven, CT, USA

E-mail: loginov@fnal.gov

We present latest results on searches for supersymmetry in final states with photons at CDF and DØ Run II in a data sample with integrated luminosity up to $4.1 \mathrm{fb}^{-1}$. We report on searches for Gauge-Mediated SUSY breaking in events with $\gamma \gamma E_{T}$ and $\gamma j E_{T}$ and a search for dark photons in events with $\gamma \ell \ell \mathrm{E}_{\mathrm{T}}$.

European Physical Society Europhysics Conference on High Energy Physics, EPS-HEP 2009, July 16 - 222009

Krakow, Poland

* Speaker.

${ }^{\dagger}$ on behalf of the CDF and DØ Collaborations 


\section{Introduction}

The Standard Model [1] of particle physics (SM) describes data from collider experiments very well [2] with no unambiguous hints of effects beyond the SM, so called "New Physics" (NP). The SM predicts the existence of $W$ and $Z$ bosons, the gluon and two of the heavier quarks. The only missing particle in the SM is the Higgs boson, which gives rise to masses of particles in the SM. The Higgs boson, unlike other SM particles, is directly sensitive to $\Lambda_{U V}$, an ultraviolet momentum cutoff, which leads to enormous quantum corrections to its mass $\left(\Delta m_{H}^{2}\right)$, and therefore it causes a serious conceptual problem. There are a few other flaws in the SM, such as prediction of massless neutrinos and inability to explain observations from non-collider experiments. For instance, astrophysical data provide a strong motivation for massive stable neutral particles, missing in the SM. In supersymmetry (SUSY) models [3], each of the SM particles has a partner differing by a half-unit of spin, which resolves the $\Delta m_{H}^{2}$ issue. If R-parity is conserved, SUSY particles are produced in pairs and their decay leads to SM particles and to the lightest SUSY particle, which is stable, and therefore is a "Dark Matter" candidate. Theoretical motivation for SUSY comes from the extrapolation of strong, electromagnetic and weak interactions to high energies, so that all the forces all coincide at the Planck scale (which is not the case for the SM). In these proceedings we present the latest CDF and D $\varnothing$ results on the searches for SUSY in a data sample with integrated luminosity up to $4.1 \mathrm{fb}^{-1}$.

\section{Physics}

High- $\mathrm{p}_{\mathrm{T}}$ photons $(\gamma)$ are copiously produced by decays of hadrons in jets resulting from scattered quarks or gluons. We take a data-driven (DD) approach to the backgrounds due to difficulties in the Monte Carlo simulation associated with modeling them, for instance the QCD (jets misidentified as photons) contributions. A short overview of procedures to estimate DD background to photon searches is presented in Ref. [4]. In these proceedings we present searches for SUSY in photon final states. We report results on searches for Gauge-Mediated SUSY breaking in events with $\gamma \gamma E_{\mathrm{T}}$ and $\gamma j \mathbb{E}_{\mathrm{T}}$ (Section 2.1) and for dark photons in events with $\gamma \ell \ell E_{\mathrm{T}}$ (Section 2.2).

\subsection{Search for Gauge-Mediated SUSY breaking in $\gamma \gamma E_{T}$}

At the Tevatron gaugino pair-production (mostly $\widetilde{\chi}_{1}^{+} \widetilde{\chi}_{1}^{-}$and $\widetilde{\chi}_{1}^{ \pm} \widetilde{\chi}_{2}^{0}$ pairs) is expected to dominate, and the cascade decays of $\widetilde{\chi}_{2}^{0}$ and $\widetilde{\chi}_{1}^{ \pm}$produce two neutralinos $\widetilde{\chi}_{1}^{0}$ in association with other particles. In a Gauge-Mediated Supersymmetry breaking (GMSB) models $\widetilde{\chi}_{1}^{0}$ almost always decays exclusively into a photon and a lightest superpartner, gravitino $(G)$, with mass of the order of $1 \mathrm{keV} / \mathrm{c}^{2}, \widetilde{\chi}_{1}^{0} \rightarrow G \gamma$. The $G$ escapes detection and results in large missing transverse energy $\left(\mathbb{E}_{\mathrm{T}}\right)$. We present the results of searches for GMSB with $\tilde{\chi}_{1}^{0} \rightarrow \gamma G$ in the $\gamma \gamma E_{\mathrm{T}}$ and $\gamma j E_{\mathrm{T}}$ final states.

In $2.6 \pm 0.2 \mathrm{fb}^{-1}$ recorded by the CDF II detector we search for GMSB with $\widetilde{\chi}_{1}^{0}$ lifetime $\tau_{\widetilde{\chi}_{1}^{0}}<2 \mathrm{~ns}$, which is favored for large neutralino masses. We observe no candidate events in $\gamma \gamma \mathrm{E}_{\mathrm{T}}$, consistent with a standard model background expectation of $1.4 \pm 0.4$ events. We set limits on the cross section at the $95 \%$ C.L. and place the world best limit of $149 \mathrm{GeV} / \mathrm{c}^{2}$ on the $\widetilde{\chi}_{1}^{0}$ mass at $\tau_{\widetilde{\chi}_{1}^{0}}=0$ ns. We also make exclusions in the $\widetilde{\chi}_{1}^{0}$ mass-lifetime plane for $\tau_{\widetilde{\chi}_{1}^{0}} \lesssim 2$ ns (Figure 1). D $\varnothing$ 

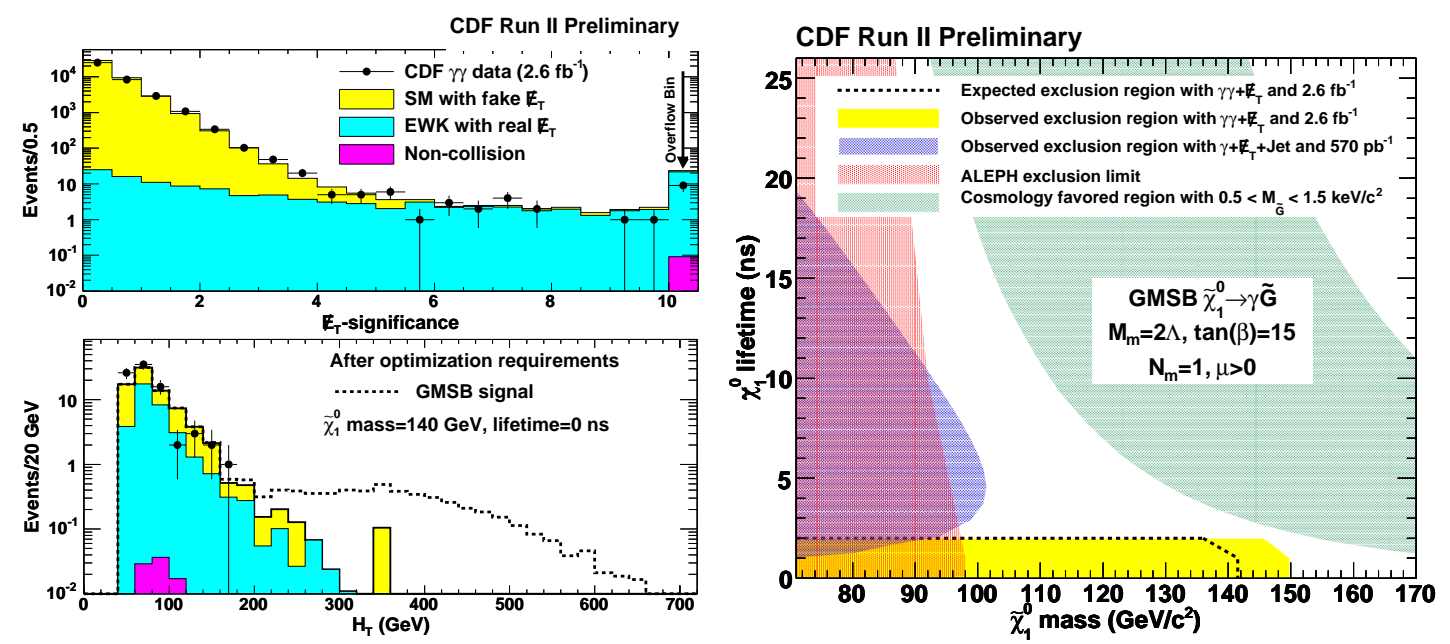

Figure 1: On the left: The $E_{T}$-significance (top) and the total transverse energy, $H_{T}$ (bottom) distributions in $\gamma \gamma \mathbb{E}_{\mathrm{T}}$ events. On the right: the predicted and observed exclusion region along with the limit from ALEPH/LEP and the $\gamma j E_{\mathrm{T}}$ delayed photon analysis [7].

assumed [5] that $\tau_{\widetilde{\chi}_{1}^{0}}$ is sufficiently short to yield decays with prompt photons, and set limits of $M_{\widetilde{\chi}_{1}^{0}}>125 \mathrm{GeV} / \mathrm{c}^{2}, M_{\widetilde{\chi}_{1}^{ \pm}}>229 \mathrm{GeV} / \mathrm{c}^{2}$ for $\tau_{\widetilde{\chi}_{1}^{0}}=0 \mathrm{~ns}$ with $1.1 \mathrm{fb}^{-1}$ of data. Exclusion limits of another CDF search for GMSB in $\gamma j \mathrm{E}_{\mathrm{T}}$ [7] in $0.6 \mathrm{fb}^{-1}$, optimized for $2 \mathrm{~ns}<\tau_{\widetilde{\chi}_{1}^{0}}<10 \mathrm{~ns}$, are also shown in Figure 1.

\subsection{Search for Dark Photons}

Hidden valley models [8] introduce a new "hidden" sector, which is very weakly coupled to the SM particles. We consider a scenario in which supersymmetric partners are pair produced and decay into SM particles and lightest neutral gaugino $\widetilde{\chi}_{1}^{0}$. The $\widetilde{\chi}_{1}^{0}$ then can decay into the hidden sector state $\widetilde{X}$ and either a photon or a new light gauge boson, a dark photon $\left(\gamma_{D}\right), \widetilde{\chi}_{1}^{0} \rightarrow \gamma \widetilde{X}$ and $\widetilde{\chi}_{1}^{0} \rightarrow \gamma_{D} \widetilde{X}$, respectively. Note that the $\widetilde{\chi}_{1}^{0} \rightarrow \gamma \widetilde{X}$ decay signature is similar to the GMSB $\widetilde{\chi}_{1}^{0} \rightarrow \gamma G$, considered in Section 2.1.

DØ performs a search for a dark photon [9]. Hadronic $\gamma_{D}$ decays are overwhelmed by SM jet backgrounds, hence, we only consider dark photon decays into isolated electron or muon pairs. Both $\widetilde{X}$ escape detection and result in large $E_{\mathrm{T}}$. We therefore investigate a previously unexplored final state that contains a photon, two spatially close leptons, and $E_{\mathrm{T}}$. To identify spatially close leptons we require $\Delta R_{\ell \ell}<0.2$ between the two leptons $\ell \ell$, and adjust isolation requirements, so that the dilepton object $\ell \ell$ is isolated. Results of the search are shown in Figure 2.

\section{Summary}

By the end of Run II both CDF and DØ expect to collect about $10 \mathrm{fb}^{-1}$ of data for analysis. Larger data sample will allow to look for rarer signatures and also to improve limits. For instance, with an integrated luminosity of approximately $10 \mathrm{fb}^{-1}$, CDF expects a $\widetilde{\chi}_{1}^{0}$ mass reach of $\approx 160 \mathrm{GeV} / \mathrm{c}^{2}$ at a $\tilde{\chi}_{1}^{0}$ lifetime of $0 \mathrm{~ns}$. New data-driven background estimation techniques, as well as new physics ideas will be useful for new searches at the Tevatron and for the LHC. 

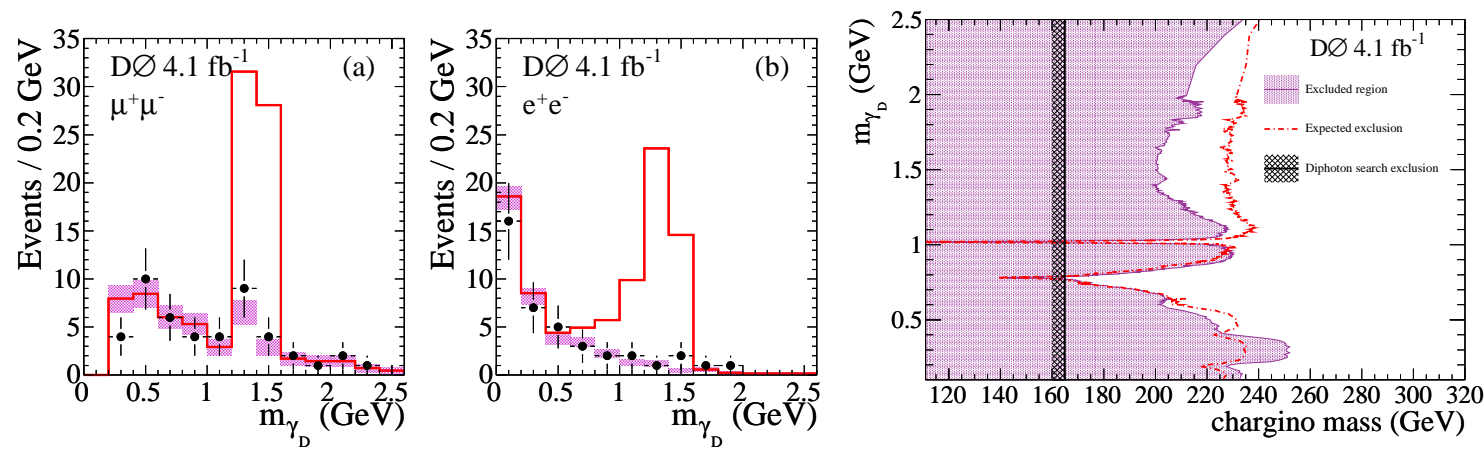

Figure 2: On the left: observed mass distributions in the signal region are represented as points with error bars, the background estimation is shown as filled band, and an example signal for $m_{\gamma D}=1.4 \mathrm{GeV}$ plus background is shown as the solid histogram for the dimuon channel (a) and the dielectron channel (b). On the right: the excluded region of possible masses of $\widetilde{\chi}_{1}^{ \pm}$and the $\gamma_{D}$ for $\operatorname{Br}\left(\widetilde{\chi}_{1}^{0} \rightarrow \gamma \widetilde{X}\right)=0.5$ are shown as the shaded region. The expected limit is illustrated as the dash-dotted line. The vertical black line corresponds to the exclusion from the diphoton search [5]. For this figure we use a convention that "mass" refers to $\mathrm{mc}^{2}$, so that it is measured in $\mathrm{GeV}$.

\section{References}

[1] S.L. Glashow, Nucl. Phys. 22, 588 (1961); S. Weinberg, Phys. Rev. Lett. 19, 1264 (1967); A. Salam, Proc. 8th Nobel Symposium, Stockholm, (1979).

[2] C. Amsler et al. (Particle Data Group), Physics Letters B667, 1 (2008).

[3] J. Wess and B. Zumino, Nucl. Phys. B 70 (1974). For reviews, see H.E. Haber and G.L. Kane, Phys. Rept. 117, 75 (1985); S.P. Martin, hep-ph/9709356 (1997).

[4] A. Loginov (for the CDF Collaboration), "CDF Searches for New Physics with Photons", Proceedings of SUSY 2007 Conference, FERMILAB-CONF-07-531-E, arXiv:0710.1964.

[5] DØ Collaboration, Phys. Lett. B 659 , 856 (2008).

[6] CDF collaboration, to be submitted to Phys. Rev. Lett.

[7] CDF collaboration, PRD 78, 0321015 (2008); CDF collaboration, Phys. Rev. Lett. 99, 121801 (2007).

[8] T. Han, Z. Si, K. Zurek, and M. Strassler, JHEP 0807, 008 (2008); M. Strassler and K. Zurek, Phys. Lett. B 651, 374 (2007).

[9] V. M. Abazov et al. (DØ Collaboration), Phys. Rev. Lett.103, 081802 (2009), arXiv:0905.1478. 